Draft Version, ACCEPted for Publication in ApJ

Preprint typeset using LTEX style emulateapj v. 5/2/11

\title{
THE PARABOLIC JET STRUCTURE IN M87 AS A MAGNETOHYDRODYNAMIC NOZZLE
}

\author{
MASANORI NAKAMURA ${ }^{1}$ AND KEIICHI ASADA ${ }^{1}$ \\ ${ }^{1}$ Institute of Astronomy \& Astrophysics, Academia Sinica, P.O. Box 23-141, Taipei 10617, Taiwan \\ nakamura@asiaa.sinica.edu.tw, asadaeasiaa.sinica.edu.tw \\ Draft Version, Accepted for Publication in ApJ
}

\begin{abstract}
The structure and dynamics of the M87 jet from sub-milli-arcsec to arcsecond scales are continuously examined. We analysed the VLBA archival data taken at 43 and $86 \mathrm{GHz}$ to measure the size of VLBI cores. Millimeter/sub-mm VLBI cores are considered as innermost jet emissions, which has been originally suggested by Blandford \& Königl. Those components fairly follow an extrapolated parabolic streamline in our previous study so that the jet has a single power-law structure with nearly five orders of magnitude in the distance starting from the vicinity of the supermassive black hole (SMBH), less than 10 Schwarzschild radius $\left(r_{\mathrm{s}}\right)$. We further inspect the jet parabolic structure as a counterpart of the magnetohydrodynamic (MHD) nozzle in order to identify the property of a bulk acceleration. We interpret that the parabolic jet consists of Poynting-flux dominated flows, powered by large amplitude, nonlinear torsional Alfvén waves. We examine the non-relativistic MHD nozzle equation in a parabolic shape. The nature of trans-fast magnetosonic flow is similar to the one of transonic solution of Parker's hydrodynamic solar wind; the jet becomes super-escape as well as super-fast magnetosonic at around $\sim 10^{3} r_{\mathrm{s}}$, while the upstream trans-Alfvénic flow speed increases linearly as a function of the distance at $\sim 10^{2}-10^{3} r_{\mathrm{s}}$. We here point out that this is the first evidence to identify these features in astrophysical jets. We propose that the M87 jet is magnetically accelerated, but thermally confined by the stratified ISM inside the sphere of gravitational influence of the SMBH potential, which may be a norm in AGN jets.
\end{abstract}

Subject headings: galaxies: active — galaxies: individual (M87) — galaxies:jets — magnetohydrodynamics (MHD) — methods: data analysis — methods: analytical

\section{INTRODUCTION}

How does the acceleration and collimation take place as well as the formation process in astrophysical jets? Although electromagnetic and/or magnetohydrodynamic (MHD) mechanisms are frequently invoked to extract energy and momentum from the compact object and/or the accretion disk (e.g., Blandford \& Znajek 1977; Blandford \& Payne 1982; Pudritz \& Norman 1983; Uchida \& Shibata 1985; Shibata \& Uchida 1986; Lovelace et al. 1987; Hevvaerts \& Norman 1989; Lovelace et al. 1991; Pelletier \& Pudritz 1992; Meier 1999, 2001), none of real astrophysical jet systems has yet been examined to confirm these theoretical properties in the quantitative manner.

M87 is one of the nearest active galaxies (16.7 Mpc; Jordán et al. 2005) that exhibit relativistic outflows. The mass of the central supermassive black hole $(\mathrm{SMBH}) M_{\bullet}$ is measured with a range $3.2 \times 10^{9} M_{\odot}($ e.g., Macchetto et al. 1997) to $6.6 \times 10^{9} M_{\odot}$ (Gebhardt et al. 2011). This largest mass gives an apparent angular size $\sim 8 \mu$ as for the Schwarzschild radius $r_{\mathrm{s}} \equiv 2 G M_{\bullet} / c^{2}$. This galaxy therefore provides a unique opportunity to study the relativistic outflow with the highest angular resolution in units of $r_{\mathrm{s}}$. Extended synchrotron emissions of one-sided jet, emerging from the nucleus, have been well-studied at multiwavelengths from radio to X-ray bands (Reid et al. 1989; Owen et al. 1989; Biretta et al. 1995; Perlman et al. 1999; Biretta et al. 1999; Junor et al. 1999; Marshall et al. 2002; Wilson \& Yang 2002; Perlman \& Wilson 2005; Harris et al. 2006; Ly et al. 2007; Kovalev et al. 2007), which cover a spatial scale $\sim 0.4$ mas -14 arcsec with the viewing angle $14^{\circ}$ (Wang \& Zhou 2009), corresponding to $2 \times 10^{2}-7 \times 10^{6} r_{\mathrm{s}}$ in de-projection.

Very recently, there are several attempts to examine the inner jet structures on M87; i) Hada et al. (2011) performed the core shift measurement by using multi-frequency, phase referencing VLBA observations, indicating that the $43 \mathrm{GHz}$ VLBI core is located at $\sim 20 r_{\mathrm{s}}$ from the central engine (presumably, the SMBH and/or accretion disk). They conclude that the measured frequency dependence of the core shift $\propto \nu^{-0.94 \pm 0.09}$ is in good agreement with a synchrotron selfabsorbed "conical" jet (Blandford \& Königl 1979; Königl 1981). ii) Asada \& Nakamura (2012, hereafter AN12) investigated the structure of the M87 jet, from milliarcsec (mas) to arcsec scales by utilizing the images taken with EVN, MERLIN, and VLBA. AN12 reveal that the jet maintains a "parabolic" shape $z \propto r^{1.73 \pm 0.05}$ in a de-projected distance of $10^{2}-10^{5} r_{\mathrm{s}}$, where $r$ is the radius of the jet emission and $z$ is the axial distance from the core. iii) Doeleman et al. (2012) conducted the Event Horizon Telescope (EHT) observation at a wavelength of 1.3 millimeter $(\mathrm{mm})$, deriving the size of $230 \mathrm{GHz}$ VLBI core to be $5.5 \pm 0.4 r_{\mathrm{s}}$. This is smaller than the diameter for the innermost stable circular orbit (ISCO) of a retrograde accretion disk, suggesting that the M87 jet may be powered by a prograde accretion disk around a spinning $\mathrm{SMBH}$.

In the standard picture of VLBI jets, the compact radio core (VLBI core) is widely believed as the throat of a diverging (with a constant opening angle), conical jet (Blandford \& Königl 1979). Alternatively, Daly \& Marscher (1988) proposed that the VLBI core is identified as the first re-collimation Mach disk - oblique shock system. The VLBI core at $\mathrm{mm}$ wavelength is located to be $10^{4-6} r_{\mathrm{s}}$ where the synchrotron emission is completely optically thin [where the optical depth $\tau(\nu)$ is fallen 
to $\sim 1$ 1, which corresponds to the turnover in the spectrum (Jorstad et al. 2007). The jet acceleration and collimation zone is speculated in the upstream of the mm-wave radio core. In the present paper, we discuss about the jet acceleration and collimation zone in M87 by introducing a parabolic jet streamline as a context of MHD nozzle, based on resent results (i) - (iii) listed above, as well as our own VLBA data reductions at $43 / 86 \mathrm{GHz}$. We apply the standard picture of the VLBI core (as an innermost jet at giving frequency) to M87, one of the most resolved AGN jets, to inspect a feasibility of the physical interpretation of the core.

The paper is organized as follows. In $\$ 2$, we give a review of observed proper motions in M87 and their interpretation. 33 summarizes a general picture of MHD jets and points to be confirmed in M87. We outline observation details in $\$ 4$ followed by our results in 55 . We analyze the jet parabolic streamline as a counterpart of the MHD nozzle in 8 . Discussions and summary are given in 87 and 8 . Throughout the paper, we use the de-projected distance along the jet (assuming the viewing angle $14^{\circ}$ ) from the central SMBH in units of $r_{\mathrm{s}}$. We note that 1 mas corresponds to $517 r_{\mathrm{s}}$ in de-projection.

\section{PROPER MOTIONS ASSOCIATED TO THE FLOW SPEED}

In a general framework on asymptotic evolutions of MHD jets in parabolic streamlines, both bulk acceleration and collimation take place simultaneously. In the present paper, we investigate the jet acceleration in order to confirm the jet acceleration and collimation zone of M87. One of the useful tools to study the jet kinematics is the direct measurement of a proper motion (an "apparent" speed $V_{\text {app }}$ of moving components) by the multi-epoch observations. Let $\theta_{\mathrm{v}}$ be a viewing angle between our line of sight and an "intrinsic" speed $V_{\text {int }}$ along the jet. Then $V_{\text {app }}$ is corrected in the units of the speed of light $c(\beta=V / c)$ through the usual relationship:

$$
\beta_{\text {app }}=\frac{\beta_{\text {int }} \sin \theta_{\mathrm{v}}}{1-\beta_{\text {int }} \cos \theta_{\mathrm{v}}} .
$$

Proper motions of the M87 jet in the parabolic region $(\lesssim$ $10^{5} r_{\mathrm{s}}$ ) have been probed by VLBI observations during the past two decades (e.g., Reid et al. 1989; Kellermann et al. 2004; Kovalev et al. 2007) as shown in Fig. 1, exhibiting a series of subluminal motions. Note that a peak of apparent motions, that is superluminal $\beta_{\mathrm{app}} \sim 6$, is located at around the HST-1 complex (Biretta et al. 1999), where the jet has a structural transition from the parabolic to conical streamline as shown in AN12. As is clearly seen in Fig. 1, it is remarkable that observed apparent speed keeps increasing by almost two orders of magnitude at $z=10^{2}-10^{4} r_{\mathrm{s}}$, where the jet structure is being parabolic.

In the literature as listed above, these subluminal motions upstream of HST-1 have been generally interpreted as stationary patterns as standing shocks and/or some modes of plasma instabilities. This may be because one could consider the M87 jet structure as a cylindrical or conical structure (e.g., Kovalev et al. 2007). When the supersonic, hydrodynamic jet, which is highly over-pressured against the ambient ISM, is causally disconnected from its driving engine, forming recollimation shock due to the pressure imbalance between the inside and outside the jet (Sanders 1983). Or perhaps, growing plasma instability and its related turbulence may be speculated. However, we suggest neither would be the case in M87. As AN12 revealed, the jet structure is parabolic so that the jet outer edge still has a causal contact with its interior, where the jet could be magnetically accelerated up to over $10^{5} r_{\mathrm{s}}$ scale.

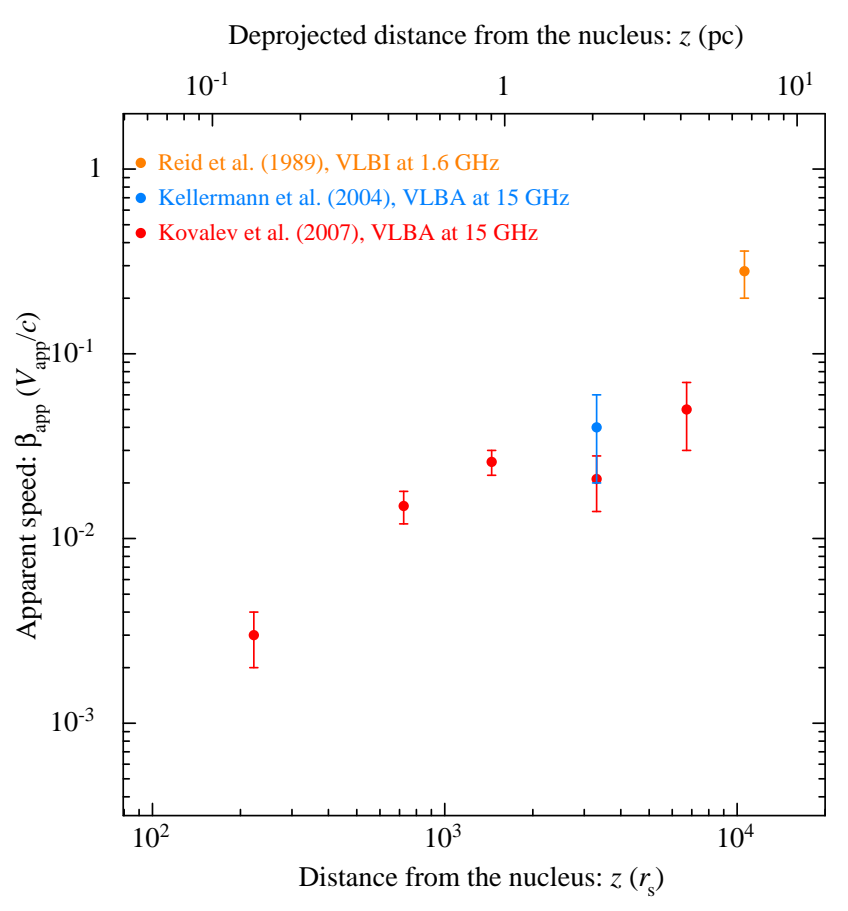

FIG. 1.- Distribution of the apparent speed $\beta_{\mathrm{app}}\left(=V_{\mathrm{app}} / c\right)$ as a function of the projected distance from the core. Measurements by VLBA at $15 \mathrm{GHz}$ (red and blue, Kovalev et al. 2007; Kellermann et al. 2004, respectively), and global VLBI at 1.6 GHz (orange, Reid et al. 1989).

Lister et al. (2009) investigate the parse-scale AGN jet kinematics in the complete MOJAVE survey; a median rms dispersion of apparent speeds in the overall sample is three times larger than that value within an individual jet. Twosided jets in relatively low-luminosity and nearby radio galaxies provide notable evidence that apparent motions in the jet and counter jet are correlated. These survey may give an additional insight that there is a characteristic flow that describes each jet; apparent motions are not simply (shock/instability) patterns, which propagate slowly and/or relatively stable, but do indeed represent underlying bulk flows. Even $V_{\text {app }}$ is corrected by a viewing angle with Eq. (1), random distribution of $V_{\text {pat }}$ at two sides should be generally expected no matter whether the jet and counter jet are intrinsically symmetric (similar kinematics of each side due to a common origin of the jet initiation). In at least NGC 1052 and Cygnus A, Lister et al. (2009) confirm a one-to-one association between features on the two sides, supporting a hypothesis that observed proper motions reflect the real jet flow. It would seem far more natural to have $V_{\text {int }}$ tied to the jet (fluid) bulk speed $V$ (Biretta et al. 1995) as

$$
\beta_{\text {int }} \lesssim \beta
$$

Another important remark is that MHD jets, which are powered by torsional Alfvén waves (TAWs) (Uchida \& Shibata 1985; (Shibata \& Uchida 1985), are intrinsically robust compared to purely hydrodynamic jets regarding nonlinear wave perturbations. When wave amplitudes are no longer small, most disturbances such as a (magneto-)sonic wave cease to propagate as a wave with a constant profile, but instead distort in time (steepening into a shock). However, Alfvén waves can continue to propagate without distortions even when their amplitudes are large (Priest 1981). Recently, this unusual, 
but very interesting property is also verified in the relativistic Alfvénic perturbations (Heyvaerts et al. 2012). We thus suggest that observed subluminal patterns in M87 may correspond to slowly, but presumably non-relativistic fluids that are magnetically accelerated along the jet parabolic streamline. We will examine this in 6

\section{GENERAL PICTURE OF MHD JETS IN GLOBAL SCALES}

We here remark one of pioneering studies in the field of MHD outflows by Blandford \& Payne (1982, hereafter BP82). Authors examined axisymmetric, steady, self-similar cold magnetohydrodynamic outflows from the Keplerian accretion disk, showing that not only the initiation of bulk outflow takes place by a "magneto-centrifugal" effect (without any support by gas pressure), but also continued bulk acceleration as well as simultaneous self-collimation can be expected in the super-Aflvénic regime by dominant toroidal (azimuthal) magnetic fields. The flow eventually becomes super fast magnetosonic [note that this "classical" fast point (CFP) is not a critical point in self-similar solutions] and a causal contact between the jet interior and its outer edges by magnetosonic waves will be maintained until the flow will pass the "modified" fast point (MFP), where the "collimation" component of the poloidal speed $V_{p} \sin \theta$ toward the jet rotating axis (where $\theta$ is the local angle between the poloidal magnetic field and any straight line drawn from the origin in spherical geometry) becomes comparable to the local fast magnetosonic speed.

The concept of the MFP has been recognized by BP82, but their MHD outflow never passes the MFP (even at large distance from the disk surface). First steady, self-similar, nonrelativistic MHD (NRMHD) outflow solutions, which pass the MFP, are introduced by Vlahakis et al. (2000). Very recently, Polko et al. (2010) successively solve steady, selfsimilar, relativistic MHD (RMHD) flow solutions beyond the MFP. Their extensive survey of the parameter space reveals the location of the MFP spans from $10^{4} r_{\mathrm{g}}$ to $10^{8} r_{\mathrm{g}}$, where $r_{\mathrm{g}} \equiv G M_{\bullet} / c^{2}$ is the gravitational radius; the location of the MFP may be sensitive to the poloidal spherical angle of the Alfvén point (AP), $\theta_{\mathrm{A}}$ (a distance $D_{\mathrm{MFP}}$ monotonically decreases from $>10^{6} r_{\mathrm{g}}$ to $\sim 10^{4} r_{\mathrm{g}}$ while $\theta_{\mathrm{A}}$ increases as $40^{\circ} \rightarrow 60^{\circ}$ ) and so-called the magnetization parameter $\sigma_{\mathrm{M}}$; Poynting-to-matter energy flux ratio ( $D_{\mathrm{MFP}}$ increases from $\sim 10^{5} r_{\mathrm{g}}$ to $\sim 10^{8} r_{\mathrm{g}}$ if $\sigma_{\mathrm{M}}$ increases as $\sim 1 \rightarrow 60$ ) (Polko et al. 2013). Both non-relativistic and relativistic solutions exhibit an asymptotic bulk acceleration until the flow reaches the MFP, where the streamline remains parabolic (see also Li et al. 1992; Vlahakis \& Königl 2003, but, note that an MFP would exist at infinity in their solutions).

In the standard process of NRMHD jet acceleration, the poloidal jet speed exceeds the local escape speed around trans-Alfvénic regime (Kudoh \& Shibata 1997; Romanova et al. 1997; Vlahakis et al. 2000). Even in the RMHD jet, this feature may be held; Polko et al. (2013)'s very recent steady, axisymmetric, semi-self-similar formulation first passes through all three singular points [the "modified" slow point (MSP), AP, and MFP]. In a relativistic framework, the gravity does not follow a self-similarity and thus none of past semi-analytical RMHD solutions in a full-selfsimilar manner could not obtain a complete solution to pass all singular points. In their fiducial solution, the location of the MSP falls within the range of a surface layer of the radiatively inefficient accretion flows (RIAFs) ( $H \sim r$ and $B_{\phi} \sim B_{p}$, where $H$ the disk scale height, $B_{\phi}$ the toroidal component, and $B_{p}$ the poloidal magnetic field components, respectively: e.g., Meier 2001). A nominal magneto-centrifugal process does take place after passing the MSP and the outflow immediately passes the AP without becoming relativistic $(\gamma=1$, where $\gamma$ is the Lorentz factor). Around the AP, the flow kinetic energy $(\gamma-1)$ overtakes the gravitational potential energy. Beyond the AP, the magnetic acceleration becomes effective, increasing the poloidal flow speed towards the relativistic regime, whereas $(\gamma-1)$ only becomes relativistic further downstream along the jet.

In this paper, we suggest observed subluminal proper motions on 0.1 to $10 \mathrm{pc}$ scales (Fig. 11) may exhibit slowly, but certainly accelerated fluid components, which can be described in the framework of NRMHD parabolic jets. McKinney (2006) discussed general relativistic MHD (GRMHD) simulation results, as the ergosphere-driven jet (Blandford \& Znajek 1977) does not fit into the observed feature in M87. Instead, he suggests that the broader emission component in the M87 jet could be due to the slow disk wind, which interacts with the surrounding ISM. We also suggest that the outermost part of the jet (even it may still be powered by the frame-dragging in the ergosphere), the flow speed remains non-relativistic $\gamma \simeq 1$ at $\sim 5 \times 10^{3} r_{\text {g }}$ although the inner part becomes relativistic $\gamma \sim 5$ at this scale. The dominant toroidal magnetic pressure at the outermost part of the jet has a pressure-matching with the ambient non-magnetized coronal (thermal) pressure shown Fig. 9 in McKinney (2006).

Another theoretical effort by Gracia et al. (2009) dealt with a steady, axisymmetric, multi-layer outflows (relativistic spine jet and non-relativistic sheath wind), nicely reproducing such observed properties of M87 as an asymptotic collimation and edge-brightened emission. Also, Komissarov et al. (2007, 2009) performed axisymmetric RMHD numerical simulations to examine the magnetic jet acceleration. Jet outer boundary is assumed to be confined by a rigid wall of adopted conical/parabolic shapes, while the poloidal fluxes inside the jet are differentially collimated toward the central axis. We are especially interested in their "differential rotation" model; they prescribe a non-uniform rotation to drive the jet at the base. $\gamma$ increases mostly in the intermediate region between the inner spine and outer sheath, on the other hand $\gamma$ remains almost unity in the outer sheath region even at the further downstream of $10^{4}-10^{6}$ scales of the jet launching $(\sim$ a radius of the event horizon).

In general, (G)RMHD jets may be covered by the slowly moving outer sheath which determines the global shape of jets under the pressure equilibrium with the ISM. Therefore, we suggest the non-relativistic bulk flow in M87 may be relevant to the edge-brightened sheath part of the jet although the inner spine part of the jet has not yet been clearly identified. As we will argue in $\$ 7.3$ a future VLBI imaging of the jet emission higher than $43 \mathrm{GHz}$ will be a crucial test whether the spine/sheath structure exists.

\section{ARCHIVAL DATA AND DATA REDUCTION}

We analysed archival data (BW088 and BJ045) of VLBA observations at 43 and $86 \mathrm{GHz}$. VLBA observation at 43 GHz was carried out on 2007 January 27 at 43.127 and 43.135 GHz using all ten stations of the VLBA, but Kitt Peak station. VLBA observation at $86 \mathrm{GHz}$ was carried out on 2007 January 17 at 86.253 and $86.269 \mathrm{GHz}$ using all stations of the VLBA, but Saint Croix and Hancock stations. Each IF has $16-\mathrm{MHz}$ bandwidth in both observations. The data were correlated at the VLBA correlator at Socorro.

An a priori amplitude calibration for each station was de- 
rived from the system temperatures measured during observations and antenna gain curve information from standard files. Opacity correction was made during amplitude calibration process using the AIPS task APCAL. Fringe fitting was performed on each IF and polarization independently using the AIPS task FRING. After delay and rate solutions were determined, the data were averaged over 12 seconds in each IF and self-calibrated using Difmap. We note that the VLBA observations were conducted with dual polarization, and we independently reduced the data at $86 \mathrm{GHz}$ at both polarizations and confirmed consistency of the obtained images.

\section{RESULTS}

We show the VLBA images at 43 and $86 \mathrm{GHz}$ in Fig. 2 Image qualities are summarized in Table 1. The bright core emission at the eastern edge of the jet and continuous jet emission are detected in the image at $43 \mathrm{GHz}$. The jet shows an edge-brightened structure as seen in the previous images (Junor et al. 1999; Kovalev et al. 2007; Ly et al. 2007; Hada et al. 2011). On the other hand, we only see the bright core emission at $86 \mathrm{GHz}$, while the jet emission is not detected. This is because of the less image sensitivity, since large telescopes are not included in our imaging. Note that previous GMVA observations show some diffuse emission of the jet at this frequency (Krichbaum et al. 2006).

We define the core with a Gaussian model that was fitted to the innermost bright region in all the images. The measured size of the VLBI core at $43\left(\right.$ core $\left._{43}\right)$ and $86 \mathrm{GHz}\left(\right.$ core $\left._{86}\right)$ were $182 \pm 53$ and $100 \pm 55 \mu$ as, corresponding to $23 \pm 7 r_{\mathrm{s}}$ and $13 \pm 7 r_{\mathrm{s}}$, respectively. The measured size of core 86 is in good agreement with $99 \pm 21 \mu$ as in the previous GMVA observation (Lee et al. 2008). Also we remark the size of the VLBI core at $230 \mathrm{GHz}\left(\right.$ core $\left._{230}\right)$ is derived by the EHT observation as $5.5 \pm 0.4 r_{\mathrm{s}}$ in the correlated flux density analysis by circular Gaussian models (Doeleman et al. 2012).

Figure 3 shows the radius of the jet as a function of the de-projected distance from the central SMBH, which is essentially similar to Fig. 2 in AN12, but additional three data points of VLBI cores (core $43,86,230)$ are included. Current innermost emissions of the M87 jet are lied at $\sim$ a few of $10^{2} r_{\mathrm{s}}$ from the central SMBH, which have been observed by $43 \mathrm{GHz}$ VLBA observations. However, if we consider a hypothesis, which has been introduced by Blandford \& Königl (1979), that the VLBI core is the most upstream of the optical thin synchrotron emission at a given frequency (thus a position of the "self-absorbed core" depends on an observed frequency: e.g., Königl 1981), we can examine the further innermost jet structure by using VLBI cores with $43 \mathrm{GHz}$ and higher frequencies.

In order to determine an offset position in the jet axial direction (from the core position to the central engine), we follow the formula in Hada et al. (2011). An absolute offset $\Delta z$ can be determined as a function of the frequency as

$$
\Delta z(\nu / \mathrm{GHz})=A \nu^{-\alpha}(\mathrm{mas}),
$$

where $\alpha=0.94 \pm 0.09$ and $A=1.40 \pm 0.16$. We therefore estimate $\Delta z(43 \mathrm{GHz})=21.2 \pm 6.2 r_{\mathrm{s}}, \Delta z(86 \mathrm{GHz})=$ $10.9 \pm 4.7 r_{\mathrm{s}}$, and $\Delta z(230 \mathrm{GHz})=4.34 \pm 2.17 r_{\mathrm{s}}$, respectively. As is shown in Fig. 3, three points of VLBI cores (core $_{43}$, core $_{86}$, and core 230 ) are plotted under our assumption that the axial offset position at infinite frequency $\Delta_{\infty}(\rightarrow 0)$ is the location where the SMBH and/or accretion disk plane exist. Note that VLBA core sizes at 5.0 and $8.4 \mathrm{GHz}$ coincide the jet width derived in VLBA observations at $43 \mathrm{GHz}$ (Hada et al., in private communications), thus it may be reasonable to interpret frequency-dependent VLBI core as an innermost synchrotron emission where the jet emissions become optically thin.

The parabolic jet seems to follow a single power-law streamline with nearly five orders of magnitude in distance. Three points of VLBI cores are not used for a fit to data points to derive a power-law index $a$ of $1.73 \pm 0.05$ in AN12. One, however, has to bear in mind the following quest; where does jet origin exist and how does its non-thermal emission is initiated at some higher frequency? It is nevertheless useful for us to examine the nature of the jet parabolic structure; how it is maintained under the stratified ISM in the dominant gravitational potential by the central SMBH.

In the following sections, based on the MHD jet theory, we analyze the bulk acceleration of the trans-Alfvénic flow and derive the approximate MHD nozzle equation of the transfast magnetosonic jet in 6 . We discuss about the formation of the parabolic streamline in M87 as well as a potential limit of exploring the innermost jet emission by using VLBI core shift measurements as we quest above in $\$ 7$.

\section{ANALYSIS OF THE PARABOLIC STREAMLINE}

\subsection{Bulk Acceleration of the Trans-Alfvénic Flow}

In order to inspect the property of the NRMHD jet acceleration in the trans-Alfvénic regime, let us follow the wave dynamics of TAWs propagating along a global poloidal magnetic field, which is originally argued by Shibata \& Uchida (1985). We consider a poloidal magnetic flux tube given by

$$
B_{p} \Sigma=\Psi=\text { constant, }
$$

where $\Sigma$ is a cross section of the flux tube and $\Psi$ is the magnetic flux. $\Sigma$ is assumed to be $\Sigma \propto r^{2}$. Under the WKB approximation, the conservation of the energy flux density of the TAW is considered through a circular (cross-sectional) area around the rotation axis in cylindrical coordinate $(r, \phi, z)$ as

$$
\frac{\rho \delta V_{\phi}^{2} V_{\mathrm{A}}}{B_{p}}=\text { constant }
$$

here, $\rho, \delta V_{\phi}$ and $V_{\mathrm{A}} \equiv B_{p} /(4 \pi \rho)^{1 / 2}$ are the gas density, azimuthal component of the velocity field (a transverse displacement against the poloidal magnetic line of force drives a TAW), and Alfvén speed, respectively. From this equation, the following relation can be obtained:

$$
\delta V_{\phi} \propto \rho^{-1 / 4}
$$

Perturbed quantities in the transverse direction satisfy

$$
\delta V_{\phi}=-\frac{\delta B_{\phi}}{\sqrt{4 \pi \rho}}
$$

which is another property of the shear Alfvén wave (Priest 1981). Thus TAWs can be characterized as an equality of ratios between poloidal and toroidal components in the magnetic field,

$$
\frac{\delta B_{\phi}}{B_{p}}=-\frac{\delta V_{\phi}}{V_{\mathrm{A}}} .
$$

Note that the wave amplitudes $\left(\delta B_{\phi}\right.$ and $\left.\delta V_{\phi}\right)$ are not necessarily small, but can be arbitrary finite. In a linear regime $\left(\left|\delta B_{\phi}\right| \ll B_{p}\right)$, a perturbation of the TAW is incompressible [only magnetic tension force $\left(\boldsymbol{B}_{p} \cdot \nabla\right) B_{\phi}$ induces the 

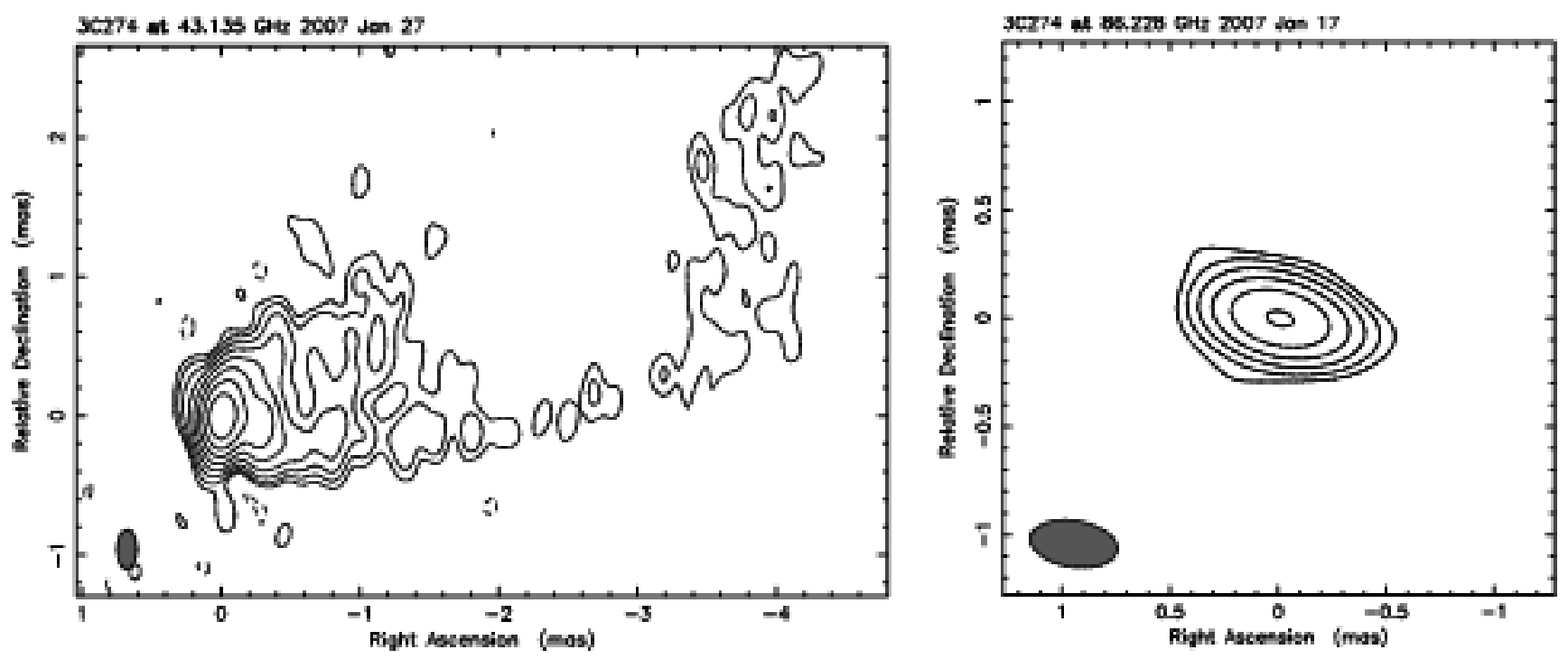

FIG. 2.- VLBA image of M87 at $43 \mathrm{GHz}$ (left). Contours are plotted at $-1,1,2, \ldots, 1024 \times 1.9 \mathrm{mJy}^{-1}$ beam $^{-1}$, which is three-times the residual r.m.s. noise. The synthesized beam is 0.281 mas $\times 0.139$ mas with the major axis at a position angle of $-0.07^{\circ}$. VLBA image of M87 at $86 \mathrm{GHz}($ right). Contours are plotted at $-1,1,2, \ldots, 1024 \times 26.3 \mathrm{mJy}_{\text {beam }}{ }^{-1}$, which is three-times the residual r.m.s. noise. The synthesized beam is 0.406 mas $\times 0.212$ mas with the major axis at a position angle of $81.6^{\circ}$

incompressible Alfvén mode], while if a wave amplitude is not negligibly small $\left|\delta B_{\phi}\right| / B_{p} \gtrsim O(1)$, the TAW also drives the compressional Alfvén mode. In a steady, axisymmetric MHD solution of BP82-type cold MHD outflow, $\left|\delta B_{\phi}\right| \simeq B_{p}$ is expected in the trans-Alfvénic flow $V_{p} \approx V_{\mathrm{A}}$ (e.g., Königl \& Pudritz 2000). On the other hand, in a noncold MHD outflow, the initial bulk acceleration takes place by the thermal pressure gradient force and then magnetic acceleration processes take over beyond the MSP. In general, steady, axisymmetric solutions exhibit $\left|\delta B_{\phi}\right| \geq B_{p}$ at around Alfvénic point in both NRMHD (Kudoh \& Shibata 1997; Vlahakis et al. 2000) and RMHD (Polko et al. 2013) regimes. Hereafter $\delta B_{\phi}$ is replaced by $B_{\phi}$ in the paper.

We therefore remark large amplitude, nonlinear TAWs play an important role in the trans-Alfvénic jet dynamics, which has been first examined in full two-dimensional MHD simulations (Uchida \& Shibata 1985; Shibata \& Uchida 1985, 1986). A magneto-centrifugal process of BP82 by quasilinear TAWs is no longer effective, while, the toroidal magnetic pressure gradient force in the poloidal direction $\nabla_{p}\left(B_{\phi}^{2} / 8 \pi\right)$ becomes dominant in the trans-Alfvénic regime. The uncoiling of $B_{\phi}$ produces a compression of the plasma in the poloidal direction, which drives fast magnetosonic waves, powering the bulk acceleration. On the other hand, the hoop stress: $-B_{\phi}^{2} / 4 \pi r$ squeezes the plasma toward the central axis. Physical picture described above is named as "sweeping magnetic twist" mechanism by Uchida \& Shibata (1986).

Considering the trans-Alfvénic NRMHD bulk flow powered by nonlinear TAWs, order-of-magnitude estimations of the one-dimensional $(z)$ equation of motion give

$$
V_{z} \sim \frac{B_{\phi}^{2}}{4 \pi \rho} V_{\mathrm{A}}^{-1} .
$$

By combining eqs. (6), (8), and (9), Shibata \& Uchida (1985) derive

$$
V_{z} \sim B_{p}^{-1}=\text { constant. }
$$

Thus, the terminal velocity of TAW-driven MHD jet model with an initially uniform axial field $\left(B_{p}=\right.$ con- stant) is a constant with an order of trans-Alfvénic speed (e.g., Shibata \& Uchida 1985; Kudoh et al. 1998; Kigure \& Shibata 2005).

On the other hand, the terminal velocity of TAW-driven MHD jet with a non-uniform axial field ( $B_{p} \neq$ constant) can be super-Alfvénic (even super-fast magnetosonic) in the downstream. We thus suggest

$$
V_{z} \propto z^{2 / a}
$$

Again, $a$ denotes the power-law index of the parabolic streamline $z \propto r^{a}(1<a \leq 2)$ of a magnetic flux tube. In the specific case of a purely parabolic stream with $a=2$, we note the trans-Alfvénic jet may exhibit that the flow speed increases linearly as a function of the distance $V_{z} \propto z$, which can be observed in NRMHD simulations (e.g., Contopoulos 1995: Ouyed \& Pudritz 1997).

In Fig. 4, we show the bulk speed $\beta(=V / c)$ corrected by Eq. (1) (again, adopting $\theta_{\mathrm{v}}=14^{\circ}$ ) under an assumption of Eq. (2) as a function of the de-projected distance, showing a feature of the acceleration from $\beta \simeq 10^{-2}$ to 0.5 in the range of $z=10^{2}-10^{4} r_{\mathrm{s}}$. A distribution of $\beta$ exhibits a linear increase as a function of $z$ as $\beta \propto z$ around $z \simeq 10^{2}$ $-10^{3} r_{\mathrm{s}}$, suggesting a trans-Alfvénic flow. In order to fit the data points and derive a solid picture, more sampling from this region is desired. Therefore, higher angular resolution and higher sensitivity observation with detailed analysis to identify more components and trace the proper motions is important. Beyond $z \gtrsim 10^{3} r_{\mathrm{s}}$, it seems that the bulk acceleration is weakened slightly, indicating that the jet may transit from trans-Alfvénic to trans-fast magnetosonic, but the underlying flow is still accelerated toward a relativistic regime $\beta \lesssim 1$.

\subsection{MHD Nozzle Equation in the Trans-magnetosonic Flow}

Of our particular interest is the trans-(fast) magnetosonic flow in the parabolic streamline. While the fraction of magnetic energy converted into bulk kinetic energy may still be small at the CFP, a further conversion from magnetic to kinetic energy and therefore a continued bulk acceleration would take place beyond the CFP via the so-called the "magnetic nozzle" effect, which has originally been pointed 


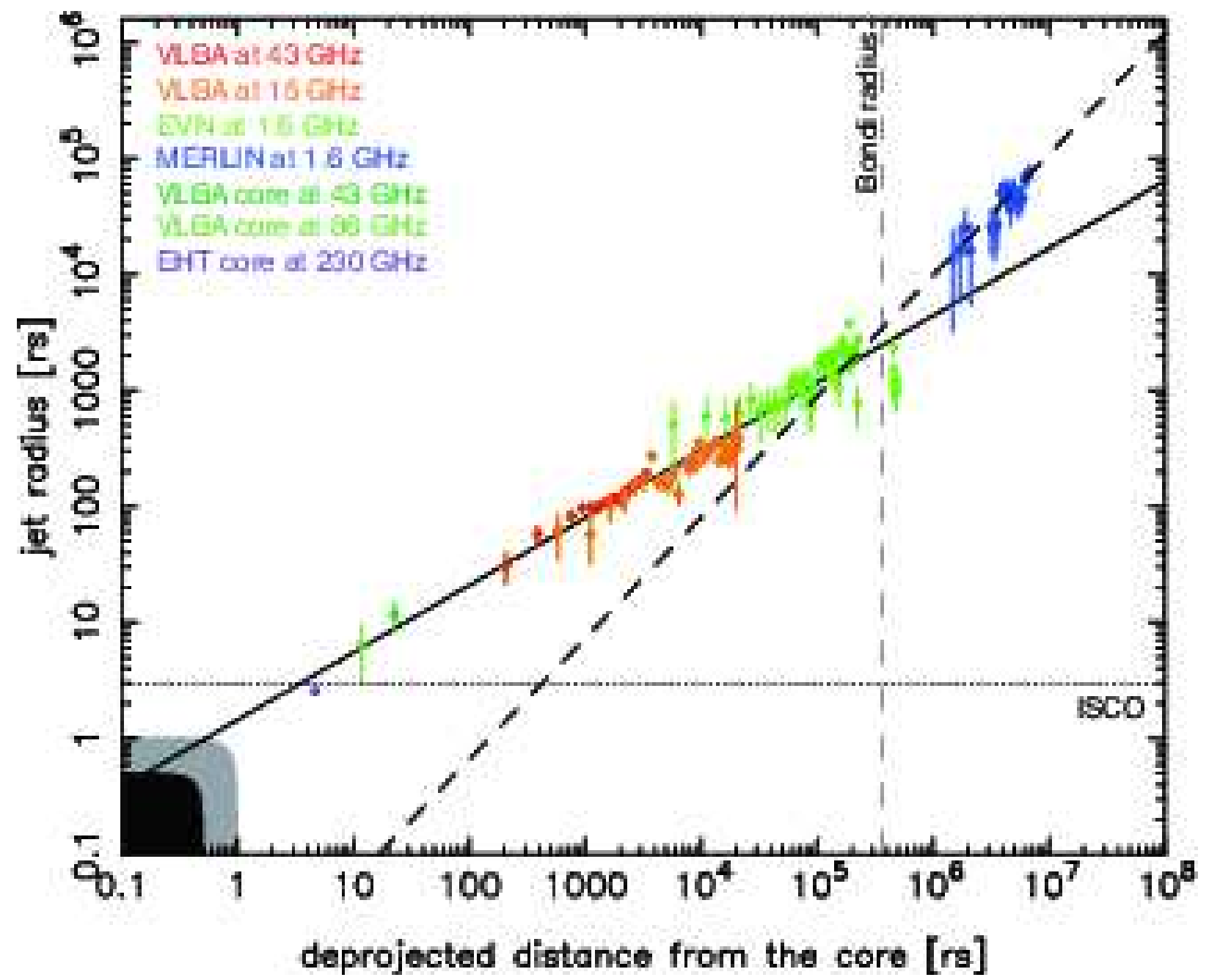

FIG. 3.- Distribution of the radius of the jet as a function of the de-projected distance from the core in units of $r_{\mathrm{s}}$. Readers can refer to Fig. 1 in Asada \& Nakamura (2012) for detailed description. Three data points of VLBI cores (at 43, 86, and $230 \mathrm{GHz}$ ) are added as the most inner jet emissions at each frequency. The solid line is the linear least-square for data points except three inner cores (VLBA at $43 / 86 \mathrm{GHz}$ and EHT at $230 \mathrm{GHz}$ ), indicating the parabolic streamline with a power-law index $a$ of $1.73 \pm 0.05$. On the other hand, the dashed line indicates the conical streamline with $a$ of $0.96 \pm 0.1$. HST- 1 is located around $5 \times 10^{5} r_{\mathrm{s}}$. The thin dashed line denotes the Bondi accretion radius located at $R_{\mathrm{B}} \simeq 3.8 \times 10^{5} r_{\mathrm{s}}$. The black area shows the size of the minor axis of the event horizon of the spinning black hole with maximum spin. The gray area indicates the size of the major axis of the event horizon of the spinning black hole with maximum spin, and corresponds to the size of the event horizon of the Schwarzschild black hole. The thin dotted line indicates the size of the inner stable circular orbit (ISCO) of the accretion disk for the Schwarzschild black hole.

out by Camenzind (1989); poloidal magnetic flux is required to diverge sufficiently rapidly in order that most of the Poynting flux in the initially magnetically dominated outflow can be converted into the kinetic energy flux beyond the CFP. This fundamentally important hypothesis has been confirmed in steady, axisymmetric solutions in both RMHD (Li et al. 1992; Li 1993; Vlahakis 2004) and NRMHD (Kudoh \& Shibata 1997) regimes.

We examine a steady $(\partial / \partial t=0)$ and axisymmetric $(\partial / \partial \phi=0)$, NRMHD outflow along the poloidal streamline in cylindrical coordinate $(z, \phi, z)$. The system of ideal MHD equations consists of the continuity, momentum (including the gravity), entropy, and Maxwell equations and Ohm's law (e.g., Lovelace et al. 1987):

$$
\begin{aligned}
& \nabla \cdot(\rho \boldsymbol{V})=0, \\
& \rho(\boldsymbol{V} \cdot \nabla) \boldsymbol{V}=-\nabla p+\frac{1}{c} \boldsymbol{J} \times \boldsymbol{B}-\rho \nabla \phi_{\mathrm{g}}, \\
& \boldsymbol{V} \cdot \nabla\left(p / \rho^{\Gamma}\right)=0,
\end{aligned}
$$

$$
\begin{aligned}
& \nabla \times \boldsymbol{B}=\frac{4 \pi}{c} \boldsymbol{J}, \nabla \times \boldsymbol{E}=0, \nabla \cdot \boldsymbol{B}=0, \\
& \boldsymbol{E}+\frac{\boldsymbol{V}}{c} \times \boldsymbol{B}=0,
\end{aligned}
$$

where $\Gamma, \phi_{\mathrm{g}}=-G M_{\bullet} /\left(r^{2}+z^{2}\right)^{1 / 2}, \boldsymbol{J}$, and $\boldsymbol{E}$ are the specific heat ratio, gravitational potential, current density, and electric field, respectively. By using eq. (12) and the solenoidal constraint in eq. (15), the velocity field can be split into poloidal and toroidal components as

$$
\boldsymbol{V}=\boldsymbol{V}_{p}+V_{\phi} \hat{\boldsymbol{e}}_{\phi},
$$

with

$$
\begin{aligned}
\boldsymbol{V}_{p} & =V_{r} \hat{\boldsymbol{e}}_{r}+V_{z} \hat{\boldsymbol{e}}_{z} \\
& =\frac{1}{4 \pi \rho r}\left(-\frac{\partial \Phi}{\partial z} \hat{\boldsymbol{e}}_{r}+\frac{\partial \Phi}{\partial r} \hat{\boldsymbol{e}}_{z}\right),
\end{aligned}
$$

where $\Phi(r, z)$ is the Stokes' poloidal stream function and constant along a streamline. 


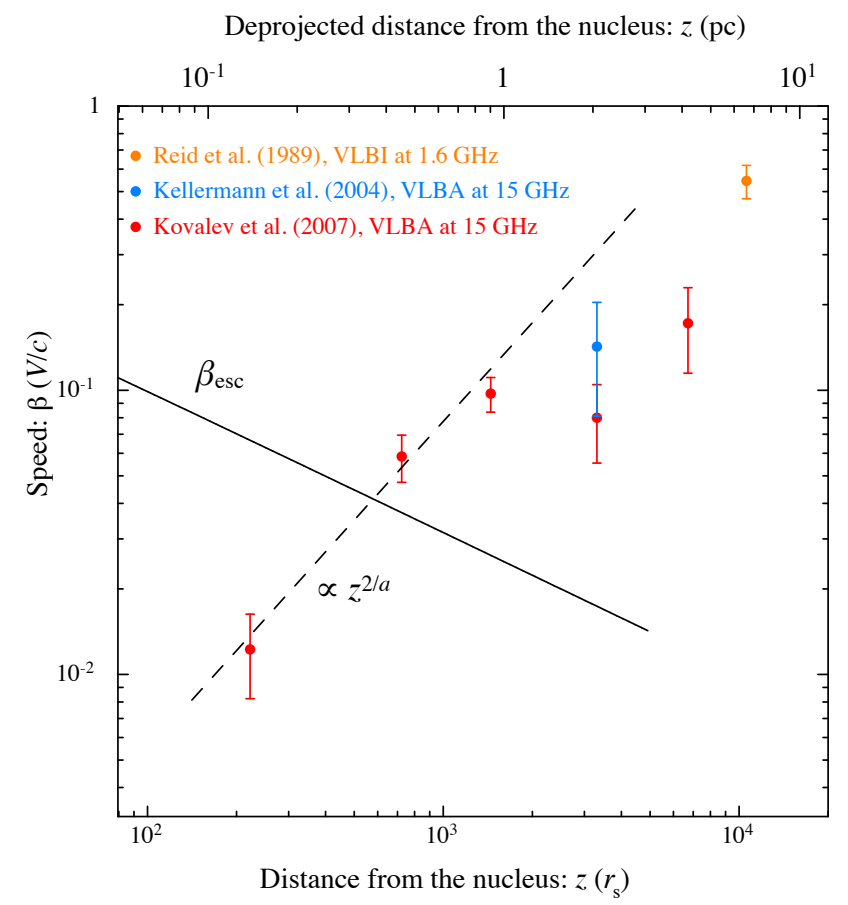

FIG. 4.- Distribution of the bulk speed $\beta(=V / c)$ as a function of the deprojected distance from the core. Measurements are illustrated in the same manner as Fig. 1 The dashed line represents $\beta \propto z^{2 / a}$ with $a=1.73$; note it is not a fit to data points, but is derived analytically in 6.1 The solid line shows the escape speed $\beta_{\text {esc }}\left(=V_{\text {esc }} / c\right)=1 / \sqrt{z}$ (normalized by $r_{\mathrm{s}}$ ) along the jet parabolic (solid) streamline shown in Fig. 3

We consider that the super-Alfvénic jet carries a substantial energy in the form of Poynting flux along the axial $(z)$ direction, especially for a limit of $B_{\phi} \gg B_{p}$ (in other words, the system can be described as a current-carrying jet, e.g., Contopoulos 1995). As is mentioned above, the divergence of poloidal magnetic flux is one of key factors to attain an efficient conversion of the Poynting flux into the kinetic energy flux. Kudoh \& Shibata (1997) investigate two cross sections of a poloidal magnetic flux $\Sigma$ in steady, axisymmetric jets; the solution for $\Sigma \propto r^{2}$ almost fails to convert the Poynting flux into the kinetic energy flux beyond the CFP so that no further bulk acceleration occurs, remaining the Poynting-flux dominated (two-thirds of the total energy) at $z / r_{0} \sim 10^{6}$, where $r_{0}$ denotes the radius at the foot point of the jet. On the other hand, if the poloidal magnetic flux expands more than the purely parabolic case beyond the Alfvén radius $\left(\Sigma \propto r^{2}+r^{3} / r_{\mathrm{A}}\right)$, the bulk acceleration continues until the Poynting flux decreases to zero, well beyond the CFP at around $z / r_{0} \simeq 10^{5}$.

$r$-dependence of $\Sigma$ defines a divergence of the poloidal magnetic flux, which is physically related to the degree of the magnetic collimation of poloidal magnetic flux by the hoop stress. Three-dimensional NRMHD simulations of currentcarrying jets exhibit the distribution of axial component of the magnetic field becomes steeper, $B_{z} \propto r^{-3}$ in superAlfvénic regime (Nakamura \& Meier 2004). In general, the inwardly directed hoop stress (magnetic tension) becomes dominant in the Lorentz forces at the inner core (spine), while the outwardly directed magnetic pressure, which is dominant in the Lorentz forces at the outer edge (sheath), will be balanced with the external pressure so that the boundary shape of jets may not be magnetically self-determined (the hoop stress plays a minor role), but be controlled by the ambient ISM (Nakamura et al. 2006) as we will also discuss in 7.1 In late years, RMHD simulations confirm the intrinsic correlation between an efficiency of bulk accelerations and non-uniform lateral expansions of poloidal magnetic flux; it is referred as "differential bunching" of field lines (Tchekhovskoy et al. 2009) or "differential collimation" (Komissarov 2011), but they are essentially same as the magnetic nozzle effect (Camenzind 1989).

An edge-brightened feature is widely confirmed in the M87 jet from VLBI to VLA scales (Reid et al. 1989; Owen et al. 1989; Junor et al. 1999; Kovalev et al. 2007; Ly et al. 2007; Hada et al. 2011; Asada \& Nakamura 2012), indicating a hollow structure. Schematic picture of our MHD nozzle model is shown in Fig. 5. The jet expands in a parabolic shape $z \propto r^{a}(1<a \leq 2)$ with a dominant axial component of the velocity field $V_{z}$. Our domain of examination is a hollow tube with a variable thickness $\delta r(z)$, which increases as a function of $z$. We assume that the unit normal to the area surface is parallel to the $z$-axis. A concept of the differential collimation means a gradual concentration of poloidal magnetic flux towards the central part of the flow; a faster collimation of the inner flux surfaces than of the outer ones. This is mathematically expressed (e.g., Komissarov 2011) as

$$
\frac{d}{d z}\left(\frac{\delta r}{r}\right)>0
$$

which is equivalent to

$$
\frac{1}{\delta r} \frac{d \delta r}{d z}>\frac{1}{r} \frac{d r}{d z}
$$

Following Contopoulos (1995) (see also, Liffman \& Siora 1997), we apply Gauss' divergence theorem to eq. (12) with a cross-sectional area $r \delta r$ :

$$
\rho V_{z} r \delta r=\text { constant }=\kappa(\Phi) \text {. }
$$

Faraday's law in Eq. (15) together with Eq. 16 implies the "frozen-in" field equation $\nabla \times(\boldsymbol{V} \times \boldsymbol{B})=0$. Using Stokes' theorem, we derive

$$
V_{z} B_{\phi} \delta r=\text { constant }=\chi(\Phi) .
$$

The entropy equation (14) gives the adiabat

$$
p / \rho^{\Gamma}=\text { constant } \equiv Q(\Phi),
$$

where above functions $\kappa, \chi$, and $Q$ of $\Phi$ are conserved quantities along a flow streamline.

Considering eq. (13) in the one-dimensional axial ( $z$ ) direction at $z \gg r$, we can use the following equation:

$$
\rho V_{z} \frac{d V_{z}}{d z}=-\frac{d p}{d z}-\frac{d}{d z}\left(\frac{B_{\phi}^{2}}{8 \pi}\right)-\rho \frac{G M_{\bullet}}{z^{2}} .
$$

By differentiating eqs. 21) - 23) and substituting them into eq. (24), we obtain

$$
\left(V_{z}^{2}-V_{f}^{2}\right) \frac{1}{V_{z}} \frac{d V_{z}}{d z}=\frac{V_{f}^{2}}{\delta r} \frac{d \delta r}{d z}+\frac{C_{s}^{2}}{r} \frac{d r}{d z}-\frac{G M_{\bullet}}{z^{2}}
$$

where $V_{f}^{2}=C_{s}^{2}+V_{\mathrm{A} \phi}^{2}$ denotes the classical fast magnetosonic speed $\left(C_{s} \equiv \Gamma p / \rho\right.$ and $V_{\mathrm{A} \phi} \equiv B_{\phi}^{2} / 4 \pi \rho$ are the sonic and toroidal Alfvén speeds). Note that we eliminate the second term in the right hand side of eq. 25) because of eq. 201 


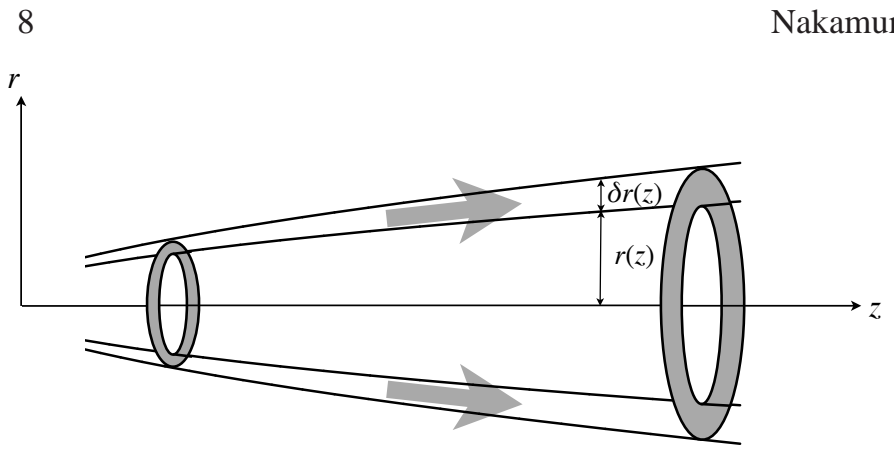

FIG. 5.- Schematic picture of our MHD nozzle model. The nozzle consists of a hollow parabolic tube, but its thickness $\delta r(z)$ increases as a function of the axial distance $z$, based on our consideration that the flow is transAlfvénic to trans-fast magnetosonic; the hoop stress differentially bunches the poloidal magnetic flux toward the central axis by the sweeping magnetic twist magnetic twist process (Uchida \& Shibata 1986)

and our assumption, $V_{f} \simeq V_{\mathrm{A} \phi}$ in the flow:

$$
\left(V_{z}^{2}-V_{f}^{2}\right) \frac{1}{V_{z}} \frac{d V_{z}}{d z}=\frac{V_{f}^{2}}{\delta r} \frac{d \delta r}{d z}-\frac{G M_{\bullet}}{z^{2}}
$$

Now supposing that $z \propto \delta r^{a}(1<a \leq 2)$, then our approximate Hugoniot equation for the parabolic MHD nozzle can be described as

$$
\frac{z}{V_{z}} \frac{d V_{z}}{d z} \approx \frac{V_{f}^{2} / a-G M_{\bullet} / z}{V_{z}^{2}-V_{f}^{2}} .
$$

Thus, for a specific case $a=2$, we speculate the poloidal jet speed exceeds the local escape speed $V_{\text {esc }}$ around trans-fast magnetosonic regime:

$$
V_{z} \approx V_{f} \approx V_{\mathrm{esc}}
$$

where $V_{\text {esc }}=\sqrt{2 G M_{\bullet} / z}$. As is discussed in Kudoh \& Shibata (1997), when a cross section expands more rapidly than $\Sigma \propto r^{2}$, the classical fast magnetosonic point approaches near the Alfvén point by an enhancement of bulk accelerations in the diverging magnetic nozzle effect, but the fast magnetosonic point is fairly bound to around to the location around $1 / 2 V_{p}^{2} \simeq \phi_{\mathrm{g}}$ in both cases $\left(\Sigma \propto r^{2}\right.$ and $\left.\Sigma_{\infty} \propto r^{3}\right)$.

Note eq. 27) represents an identical formula (when $a=2$ ) with the equation of the hydrodynamic solar wind (Parker 1958) with replacing $C_{s}$ with $V_{f}$ (the thermal pressure is substituted by the toroidal magnetic pressure). Thus, the nature of the trans-fast magnetosonic flow in a parabolic MHD nozzle, which is powered by TAWs, is similar to the one of transonic solution of Parker's solar wind. The hoop stress differentially bunches the poloidal magnetic flux toward the central axis so that a rapid expansion of the cross sectional area of the poloidal magnetic flux operates on the hollow parabolic MHD stream; we speculate a de Laval-like (Dessler 1967) diverging nozzle effect in the super-fast magnetosonic regime (see, also Fig. 5].

The distribution of $V_{\text {esc }}$ along the parabolic streamline of the M87 jet is drawn in Fig. 4 showing the location where the jet is trans-escape as well as trans-fast magnetosonic. It is notable that the jet obtains a speed of $\sim 0.04 c$ in order to escape under control of the gravity of the SMBH at around $0.2-0.3 \mathrm{pc}\left(\simeq 6 \times 10^{2} r_{\mathrm{s}}\right)$. Thus, we speculate, as a consequence of the energy conversion from Poynting to kinetic energy fluxes, the MHD bulk acceleration takes place beyond a super-fast magnetosonic regime; the magnetic nozzle effect (Camenzind 1989) is being activate by the sweeping mag- netic twist mechanism (Uchida \& Shibata 1986) so that a further energy conversion from Poynting to kinetic energy fluxes can be expected in the parabolic MHD nozzle as examined above.

If the jet is highly magnetized $\left(V_{\mathrm{A} \phi}^{2} \gg C_{s}^{2}\right)$ and/or a cross section of the poloidal magnetic flux expands more rapidly than $\Sigma \propto r^{2}$, then a position of the classical fast magnetosonic point approaches near the Alfvén point as mentioned above. This could be the case in the M87 jet. Thus, we may not distinguish clearly the trans-Alfvénic region from the transmagnetosonic region in Fig. 4. However, based on an intrinsic nature of MHD outflows, the accelerated flow first becomes trans-Alfvénic, and then trans-magnetosonic in the dynamical evolution. Note that the distribution of the bulk speed in Fig. 4 may indicate a transition from trans-Alfvénic to transmagnetosonic regime at around $\sim 10^{3} r_{\mathrm{s}}$, where the speed becomes comparable to the local escape speed. A sequential MHD bulk acceleration, which can be also powered by the toroidal magnetic pressure gradient, maintains even after weakening of the acceleration at super-Alfvénic regime as we examine the magnetic nozzle effect above.

\section{DISCUSSIONS}

\subsection{Formation of the Parabolic Streamline}

It is widely believed that AGN jets are highly supersonic from their earlier evolution stage nearby the central engine with being a narrowly beamed conical structure. Because the jet interior is out of causal contact with its outer edges by sonic waves in the lateral direction, a freely expanding ballistic flow is considered. We, however, suggested that the M87 jet is not a case of the conical jet, but the parabolic jet up to over $10^{5} r_{\mathrm{s}}$ (AN12). Furthermore, if we consider MHD jets in general, which is one of popular theoretical framework to organize the jet dynamics, it may be the norm that AGN jets are still in causal contact in the lateral expansion through a communication by magnetosonic waves.

MHD jets can be a parabolic $z \propto r^{a}(1<a \leq 2)$ if the ISM pressure is decreasing as $p_{\text {ism }} \propto z^{-b}$ with $b \simeq$ 2 (e.g., Tchekhovskov et al. 2008; Zakamska et al. 2008; Komissarov et al. 2009; Lyubarsky 2010). On the other hand, the jet boundary does not adjust to the ambient ISM pressure profile, instead asymptote to a conical shape for $b>2$ (e.g., Zakamska et al. 2008; Komissarov et al. 2009; Lyubarsky 2010; Kohler \& Begelman 2012). This indicates that the jet lateral expansion time becomes shorter than the signal propagation time of magnetosonic waves across the bulk flow, leading to a loss of causal contact to form a freely expanding ballistic jet. Non-conical, parabolic streamlines require a transverse pressure support by the extremal ISM (Blandford \& Rees 1974; Blandford \& Königl 1979). Thus, we suggest that the M87 jet may be magnetically accelerated, but thermally confined by the ISM (rather than the selfconfined in the MHD jet dynamics) as we will discuss below.

A power-law like distribution of the ISM pressure with $b \sim 2$ would be expected towards the ISM environment of M87 inside the Bondi accretion radius $R \lesssim R_{\mathrm{B}}(R$ denote the in a spherical geometry) as a consequence of hot RIAFs, which are generally considered in low luminosity AGNs. During past several decades, various type of RIAF models has been examined and we can now categorized them into three main models; the advection-dominated accretion flow (ADAF, e.g., Ichimaru 1977; Narayan \& Yi 1994), the convection-dominated accretion flow (CDAF, e.g., 
Narayan et al. 2000; Quataert \& Gruzinov 2000), and the advection-dominated inflow-outflow solution (ADIOS, e.g., Blandford \& Begelman 1999, 2004).

Given the self-similar analytical treatment among these RIAF models, the gas density distribution can be described as a power-law scaling $\rho_{\text {ism }} \propto R^{-k}$, where $k=3 / 2$ for Bondi (1952) accretion flow (BAF) and ADAF, in which the gas undergoes a nearly free-fall. On the other hand, $k=1 / 2$ in CDAF is found because the radial convection transports the angular momentum inwards, causing a reduction of the mass accretion rate and a much flatter radial density profile. ADIOS model is known to have intermediate values $1 / 2<k<3 / 2$ by taking into account a wind carrying away the mass, angular momentum, and energy. Assuming the polytropic equation of state, $p_{\text {ism }} \propto z^{-b}=R^{-(k+1)}$ (we here denote $z=R$ ). Thus, $1.5 \leq b \leq 2.5$ are allowed in RIAF models.

A recent analytical model for a giant ADAF (Narayan \& Fabian 2011) shows $b \sim 2.3$ from beyond $R_{\mathrm{B}} \simeq 5 \times 10^{5} r_{\mathrm{g}}$ down to the $\mathrm{SMBH}$, and it is universally derived for slow rotation of the ISM $(\lesssim 30 \%$ of the Keplerian speed) with the wider range of the $\alpha$-viscosity parameter (0.001 - 0.3, Shakura \& Sunyaev 1973). A spherically symmetric accretion with MHD turbulence (Shcherbakov 2008) produces $b \sim 2.25$ without a strong dependence on the parameters and the equation of state. On the other hand, thanks to comprehensive studies of numerical simulations over last decade by many authors for exploring nonlinear dynamics on RIAFs, we learn that the density profile flattens compared to the original BAF/ADAF analytical solutions, but the system does not approach a full CDAF, staying at the intermediate ADIOS, $\rho_{\text {ism }} \propto R^{-k}$ with $k \lesssim 1$, corresponding to $p_{\text {ism }} \propto z^{-b}$ with $b \lesssim 2$ (e.g., Yuan et al. 2012a b, for recent progresses on numerical simulations of RIAFs and useful references therein).

Spatial resolutions towards M87 in current X-ray instruments may not be adequate enough to resolve the region $\left(\lesssim 1^{\prime \prime}\right)$ inside the Bondi accretion radius of $3.8 \times 10^{5} r_{\mathrm{s}}$ $(\sim 250 \mathrm{pc})$ although Chandra X-ray observations confirmed ISM properties, such as the King core radius $r_{\mathrm{c}} \simeq 1.4 \mathrm{kpc}$ and the Bondi radius $R_{\mathrm{B}} \sim 250 \mathrm{pc}$ (see, e.g., Young et al. 2002; Di Matteo et al. 2003). We, however, remark that Chandra X-ray observations revealed the RIAF-like feature in a nearby low luminosity AGN, NGC 3115 (Wong et al. 2011). Stellar kinematics of the bulge of NGC 3115 give the SMBH mass estimation as $M_{\bullet} \sim(1-2) \times 10^{9} M_{\odot}$, identifying this source to be the nearest $>10^{9} M_{\odot}$ SMBH because of its proximity $D \sim 9.7 \mathrm{Mpc}$ Wong et al. 2011, and references therein). The Bondi radius $\left(R_{\mathrm{B}} \sim 4^{\prime \prime}-5^{\prime \prime}\right)$ is spatially resolved for the first time in AGNs; the radial density profile within $4^{\prime \prime}$ is described as $\rho_{\text {ism }} \propto R^{-1.03_{-0.21}^{+0.23}}$, giving a nice agreement with the RIAF model. Thus, we speculate that $p_{\text {ism }} \propto z^{-b}$ with $b \sim 2$ may be moderately reasonable.

\subsection{A Nature of the upstream flow within $100 r_{\mathrm{s}}$}

We first discuss about the hot accretion disk as an origin of the MHD outflow in M87. In ADAF (RIAF) model $\left(C_{s} \sim\right.$ $V_{\mathrm{K}}$, where the Keplerian speed $V_{\mathrm{K}}$ is equal to $\left.c / \sqrt{2 r / r_{\mathrm{s}}}\right)$, the disk (ion) temperature is considered as nearly virial $T_{\mathrm{i}}=$ $5.4 \times 10^{12}\left(r / r_{\mathrm{s}}\right)^{-1} \mathrm{~K}$ (Narayan \& Yi 1994). Given $r \sim 10 r_{\mathrm{s}}$ in Fig. 3 (where VLBI cores are located), the sound speed $C_{s} \simeq 0.28 c$ is also comparable to the local escape speed $V_{\text {esc }}=c / \sqrt{r / r_{\mathrm{s}}} \sim 0.3 c$. Therefore, we would seek much faster motions in the downstream than measured speeds $0.01 \mathrm{c}$ $-0.1 c$ at $10^{2}-10^{3} r_{\mathrm{s}}$ based on over a ten-year VLBA observations at $15 \mathrm{GHz}$ (Kovalev et al. 2007, see also Fig. 4), which are considered as the bulk speed in 82 If the M87 jet is indeed originated from the surface of RIAFs $\left(B_{\phi} \sim B_{p} ;\right.$ e.g. Meier 2001), as referring Polko et al. (2013) in $\$ 3$, we may not expect a standard magneto-centrifugal acceleration process (BP82) in the sub-Alfvénic region, but favor an initially trans-Aflvénic flow (see also, Contopoulos 1995). Thus, this may give some constraint on the required MHD jet model with an initial jet speed with an order of $\sim 0.1$ I at the jet footpoint.

Next, we discuss about the Kerr black hole as an origin of the MHD outflow in M87. As we introduced in $\$ 3$, the outermost sheath of the ergosphere-driven jet exhibits nonrelativistic motion $\gamma \simeq 1$ at $\sim 5 \times 10^{3} r_{\mathrm{g}}$ as seen GRMHD simulations (McKinney 2006); from a polar angle crosssectional distribution of the gas density, we find that the outermost sheath is much higher mass-loading (about four orders of magnitude) than the innermost jet spine. This may cause an inefficient bulk acceleration at least up to this spatial scale. A matter piling up at the outer surface of the jet may also be responsible for observed edge-brightened features in M87. The location of the CFP is about a few of $100 r_{\mathrm{s}}$ near the surface layer of the ergosphere-driven jet where the jet is still Poynting-flux dominated. Thus, we may speculate a behavior of the jet at sub- to trans-Alfvénic region $z \lesssim 100 r_{\mathrm{s}}$ as a magneto-centrifugal driving process (by not a disk rotation, but an ergosphere) similar to BP82 and then the jet seems to be powered by TAWs (the toroidal magnetic pressure gradient force) at trans-Alfvénic to trans-fast magnetosonic regions. Note that a magneto-centrifugal driving allows the flow to start at a very slow speed $V_{0}$ compared to the rotational motion of the magnetic field (Contopoulos 1995). In the case of non-relativistic Keplerian disk, $V_{\mathrm{K}} / V_{0} \simeq 300$ can be obtained in steady, axisymmetric NRMHD outflow solutions (Kudoh \& Shibata 1997; Vlahakis et al. 2000). Thus, even relativistic field rotations with $0.1 c-c$ nearby the black hole, we may speculate an initial jet speed with $V_{0} \ll 0.1 c$ at the jet footpoint.

One of crucial tests to constrain a nature of the upstream flow within $100 r_{\mathrm{s}}$ is to image extended jet emissions from the VLBI core. We are conducting new GMVA observations at $86 \mathrm{GHz}$ in order to resolve innermost jet structure at a few of $10^{1-2} r_{\mathrm{s}}$ scales.

\subsection{An Offset of the Jet Foot Point from the Black Hole and Effect on Frequency Depending Core Shift Measurements}

In $\$ 5$, we show three additional data points of VLBI cores seem to fairly follow a parabolic streamline derived by AN12, implying that the jet maintains a single non-conical structure with nearly five orders of magnitude in distance. We here discuss about limitations of a frequency depending VLBI core shift measurement to seek the true origin of the M87 jet. The median values of the VLBI core shifts in AGN jets are reported as 0.080 mas (Sokolovsky et al. 2011) and

\footnotetext{
${ }^{1}$ Ly et al. (2007) reported possible, but transient features $V_{\text {app }}=(0.25$ $0.40) c$ located at about 3 mas based on just two of five total epochs ( $\sim$ 8 months apart) of VLBA and global VLBI observations at $43 \mathrm{GHz}$; these components would not be present in other epochs. It may be still unclear whether a detection of an order of $V=0.5 c-0.6 c$ (at $\simeq 1.5 \times 10^{3} r_{\mathrm{s}}$ in de-projection) will support a RIAF-originated jet scenario.
} 
0.128 (Pushkarev 2012) between $15.4-8.1 \mathrm{GHz}$. A scale of $\sim 0.1$ mas corresponds to $1-10 \mathrm{pc}$ in de-projection for typical blazars with a small viewing angle $\theta_{\mathrm{v}} \sim 5^{\circ}$, which is equivalent to $10^{3-4} r_{\mathrm{s}}$ with $M_{\bullet}=10^{9} M_{\odot}$. Therefore, if one applies this method to blazars at even higher frequencies, an axial offset between the position of the SMBH (the origin) and/or accretion disk (the equatorial plane) and the jet foot point may be ignorable.

On the other hand, in the case of M87, the value of the VLBI core shift between $15.4-8.4 \mathrm{GHz}$ is similar $(\sim 0.1$ mas; Hada et al. 2011), but it corresponds to $\sim 10 r_{\mathrm{s}}$, approaching a limit to apply the method of frequency depending VLBI core shift measurements. By using eq. (3), we derive $\Delta z(230 \mathrm{GHz})=4.3 \pm 2.2 r_{\mathrm{s}}$, which may be comparable to the so-called stagnation surface (SS) seen in general relativistic MHD simulations (e.g., McKinney 2006). If we consider the MHD jet launching from the rotating SMBH magnetosphere (Blandford \& Znajek 1977), the SS (where the poloidal velocity component changes the sign $-/+$; the inflow onto the SMBH inside the SS, while the outflow as a jet outside the SS) has an offset from the SMBH. The location of the SS depends on a spin of the black hole; the balance between the gravity and the centrifugal forces along a poloidal magnetic field in the co-rotating frame with the line of force.

McKinney (2006) prescribes a black hole spin of $j=$ 0.9375 and the SS is located at around $2-3 r_{\mathrm{s}}$. A lower spin will put the radius of the SS further downstream $>\Delta z$ $(230 \mathrm{GHz})$. Even if we consider that the MHD jet originates from a RIAF-type accretion disk $H \sim r, r \gtrsim 2.5 r_{\mathrm{s}}$ (the lensed ISCO radius for the prograde spinning $j=1$; Doeleman et al. 2012), then the disk surface is presumably the SS so that the situation will be unchanged. Thus it may be challenging to explore the jet foot point in M87 by using VLBI cores at higher frequencies. If we take an offset of the SS into consideration, three VLBI cores may not be located on a parabolic streamline in Fig. 3. Thus, future sub-mm VLBI imaging of the jet foot point together with the SMBH shadow is crucial.

\section{SUMMARY}

Following Asada \& Nakamura (2012), we further investigate the parabolic jet structure in M87 with additional data points of VLBI cores and examine theoretical aspects of MHD jets. We here propose a hypothesis that the observed subluminal motions on $0.1-10 \mathrm{pc}$ scales in projection (corresponding to $10^{2}-10^{4} r_{\mathrm{s}}$ ) show the MHD bulk acceleration in a nonrelativistic regime although they have been interpreted during past decades as standing shocks and/or some patterns of plasma instabilities in supersonic jets. Based on our analysis, it is reasonably described as a consequence of Poynting flux-dominated flows, powered by large amplitude, nonlinear torsional Alfvén waves in a parabolic streamline. We examine the non-relativistic MHD nozzle equation in a parabolic shape. The nature of trans-fast magnetosonic flow is similar to the one of transonic solution of Parker's hydrodynamic solar wind; the jet becomes super-escape as well as super-fast magnetosonic at around $\sim 10^{3} r_{\mathrm{s}}$, while the upstream transAlfvenic flow speed increases linearly as a function of the distance at $\sim 10^{2}-10^{3} r_{\mathrm{s}}$. We here point out that this is the first evidence to identify these features in astrophysical jets.

We discuss about a formation of the parabolic streamline as a consequence of the lateral force balance between the jet internal (magnetic) and the external ISM (thermal) pressures inside the sphere of gravitational influence of the SMBH potential. An origin of the M87 jet is also discussed based on our current understanding in observations and theories although we will need further observational constraints of a nature of the upstream flow within $100 r_{\mathrm{s}}$. We furthermore suggest that it may be challenging for a seeking the true jet origin only by using VLBI core shift measurements in M87. Thus, we encourage future observations with a high sensitivity such as a space VLBI and/or mm/sub-mm VLBI at $86 \mathrm{GHz}$ or higher. In the forthcoming paper, we will examine a continued bulk acceleration from non-relativistic to fully relativistic regime, which may occur at around $10 \mathrm{pc}\left(\sim 10^{4} r_{\mathrm{s}}\right)$ or further downstream toward the HST-1 complex so that the we can complete the analysis of the parabolic jet stream line in M87 in $10^{2}-$ $10^{5} r_{\mathrm{s}}$ in a framework of MHD jets.

We appreciate an anonymous referee for critical, but very helpful comments for revising the manuscript. We acknowledge A. Doi, M. Kino, H. Nagai, and K. Hada, for useful comments and suggestions. MN thanks D. L. Meier and C. A. Norman for stimulating discussions on the MHD jet theory and Z.-Y. Li for giving useful comments on the magnetic nozzle effect in the manuscript. We are grateful to $\mathrm{M}$. Inoue, P. T. P. Ho, S. Matsushita, and our members on the Greenland Telescope project of the Academia Sinica, Institute of Astronomy and Astrophysics for their warm encouragements.

\section{REFERENCES}

Achterberg, B., Blandford, R. D., \& Goldreich, P. 1983, Nature, 304, 607

Asada, K., \& Nakamura, M., 2012, ApJ, 745, L28 (AN12)

Biretta, J. A., Zhou, F., \& Owen, F. N. 1995, ApJ, 447, 582

Biretta, J. A., Sparks, W. B., \& Macchetto, F. 1999, ApJ, 520, 621

Blandford, R. D., \& Begelman M. C. (1999), MNRAS, 303, L1

Blandford, R. D., \& Begelman M. C. (2004), MNRAS, 349, 68

Blandford, R. D., \& Königl, A. 1979, ApJ, 232, 34

Blandford, R. D., \& Payne, D. G. 1982, MNRAS, 199, 883 (BP82)

Blandford, R. D., \& Rees, M. J. 1974, MNRAS, 169, 39

Blandford, R. D., \& Znajek, R. L. 1977, MNRAS, 179, 433

Bondi, H. 1952, MNRAS, 112, 195

Camenzind, M. 1989, in Accretion Disks and Magnetic Fields in

Astrophysics, ed. G. Belvedere (Cordrecht: Kluwer), 129

Cheung, C. C., Harris, D. E., \& Stawarz, Ł. 2007, ApJ, 663, L65

Contopoulos, J. 1995, ApJ, 450, 617

Daly, R. A., \& Marscher, A. P. 1988, ApJ, 334, 539

Dessler, A. J. 1967, Rev. Geophys. 5, 1

de Matteo, T., Allen, S. W., Fabian, A. C., Wilson, A. S., \& Young, A. J.

2003, ApJ, 582, 133

Doeleman, S. S., et al. 2012, Science, 338, 355
Gebhardt, K., Adams, J., Richstone, D., et al. 2011, ApJ, 729, 119

Gracia, J., Vlahakis, N., Agudo, I., Tsinganos, K., \& Bogovalov, S. V. 2009, ApJ, 695, 503

Hada, K., et al. 2011, Nature, 477, 185

Harris, D. E., Cheung, C. C., Biretta, J. A., Sparks, W. B., Junor, W.,

Perlman, E. S., \& Wilson, A. S. 2006, ApJ, 640, 211

Heyvaerts, J., \& Norman, C. A. 1989, ApJ, 347, 1055

Heybaerts, J., Lehner, T., \& Mottez, F. 2012, A\&A, 542, A128

Ichimaru, S. 1977, ApJ, 214, 840

Junor, W., Biretta, J. A., \& Livio, M. 1999, Nature, 401, 891

Jordán, A., Côté, P., Blakeslee, J. P., et al. 2005, ApJ, 634, 1002

Jorstad, S. G., et al. 2007, AJ, 134, 799

Kellermann, K. I., et al. 2004, ApJ, 539, 563

Kigure, H., \& Shibata, K. 2005, ApJ, 634, 879

Kohler, S., \& Begelman, M. C. 2012, MNRAS, 426, 595

Kormendy, J., et al. 1996, ApJ, 459, L57

Komissarov, S. S., Barkov, M. V., Vlahakis, N., \& Königl, A. 2007, MNRAS, 380, 51

Komissarov, S. S., Vlahakis, N., Königl, A., Barkov, M. V. 2009, MNRAS, 394,1182 
TABLE 1

QUALITIES OF THE IMAGES

\begin{tabular}{lcccccc}
\hline \hline $\begin{array}{l}\text { Frequency } \\
(\mathrm{GHz})\end{array}$ & (mas) & $\begin{array}{c}\text { Synthesised Beam } \\
(\mathrm{mas})\end{array}$ & $\begin{array}{c}\text { Peak Intensity } \\
(\text { degree })\end{array}$ & $\begin{array}{c}\text { rms Noise Level } \\
\left(\mathrm{Jy} \mathrm{beam}^{-1}\right)\end{array}$ & $\begin{array}{c}\text { Dynamic Range } \\
\left(\mathrm{mJym}^{-1}\right)\end{array}$ \\
\hline 43 & 0.281 & 0.139 & $-0.07^{\circ}$ & 0.463 & 0.63 & 735 \\
86 & 0.406 & 0.212 & $81.6^{\circ}$ & 0.884 & 8.73 & 101 \\
\hline
\end{tabular}

Komissarov, S. S. 2011, Mem. Soc. Astron. Ital., 82, 95

Königl, A. \& Pudritz, R. E. 2000, Protostars and Planets IV, ed. V. Mannings et al. (Tucson, AZ: Univ. Arizona Press), 759

Kovalev, Y. Y., Lister, M. L., Homan, D. C., \& Kellermann, K. I. 2007, ApJ, 668, L27

Königl, A. 1981, ApJ, 243, 700

Krichbaum, T. P., et al. 2006, J. Phys. Conf. Ser., 54, 328

Kudoh, T., \& Shibata, K. 1997, ApJ, 474, 362

Kudoh, T., Matsumoto, R., \& Shibata, K. 1998, ApJ, 508, 186

Lamers, H. J. G. L. M., \& Cassinelli, J. P. 1999, Introduction to Stellar Wind (Cambridge: Cambridge Univ. Press)

Lee, S.-S., et al. 2008, AJ, 136, 159

Li, Z.-Y., Chiueh, T., \& Begelman, M. C., 1992, ApJ, 394, 459

Li, Z.-Y. 1993, ApJ, 415, 118

Liffman, K., \& Siora, A. 1997, MNRAS, 290, 629

Lister, M., et al. 2009, AJ, 138, 1874

Lovelace, R. V. E., Wang, J. C. L., \& Sulkanen, M. E. 1987, ApJ, 315, 504

Lovelace, R. V. E., Berk, H. L., \& Contopoulos, J. 1991, ApJ, 379, 696

Ly, C., Walker, R. C., \& Junor, W. 2007, ApJ, 660, 200

Lyubarsky, Y. 2010, MNRAS, 402, 353

Macchetto, F., Marconi, A., Axon, D. J., et al. 1997, ApJ, 489, 579

Marshall, H. L., Miller, B. P., Davis, D. S., Perlman, E. S., Wise, M.,

Canizares, C. R., \& Harris, D. E. 2002, ApJ, 564, 683

McKinney, J. C. 2006, MNRAS, 368, 1561

Meier, D. L. 1999, ApJ, 522, 753

Meier, D. L., Koide, S., \& Uchida, Y. 2001, Science, 291, 84

Meier, D. L. 2001, ApJ, 548, L9

Nakamura, M., \& Meier, D. L. 2004, ApJ, 617, 123

Nakamura, M., Li, H., \& Li, S. 2006, ApJ, 652, 1059

Narayan, R., \& Yi, I. 1994, ApJ, 428, 13L

Narayan, R., Igumenshchev, I. V., \& Abramowicz, M. A. 2000, ApJ, 539, 798

Narayan, R., \& Fabian A. C. 2011, MNRAS, 415, 3721

Ouyed, R., \& Pudritz, R. E. 1997, ApJ, 482, 712

Owen, F. N., Hardee, P. E., \& Cornwell, T. J. 1989, ApJ, 340, 698

Parker, E. N. 1958, ApJ, 128, 664

Pelletier, G., \& Pudritz, R. E. 1992, 394, 117
Perlman, E. S., Biretta, J. A., Zhou, F., Sparks, W. B., \& Macchetto, F. D. 1999, AJ, 117, 2185

Perlman, E. S., \& Wilson, A. S. 2005, ApJ, 627, 140

Polko, P., Meier, D. L., \& Markoff, S. 2010, ApJ, 723, 1343

Polko, P., Meier, D. L., \& Markoff, S. 2013, MNRAS, 428, 587

Priest, E. R. 1981, Solar Magneto-hydrodynamics (Peidel, Dordrecht), p. 160

Pudritz, R. E., \& Norman, C. A. 1983, ApJ, 274, 677

Pushkarev, A. B., et al. 2012, A\&A, 545, A113

Quataert, E., \& Gruzinov, A. 2000, ApJ, 545, 842

Reid, M. J., Biretta, J. A., Junor, W., Muxlow, T., \& Spencer, R. ApJ, 336, 112

Romanova, M. M., Ustyugova, G. V. U., Koldoba, A. V., Chechetkin,

V. M. C., \& Lovelace, R. V. E. 1997, ApJ, 482, 708

Sanders, R. H. 1983, ApJ, 266, 73

Shcherbakov, R. V. 2008, ApJS, 177, 493j

Shakura, N. I., \& Sunyaev R. A, 1973, ARA\&A, 24, 337

Shibata, K., \& Uchida, Y. 1985, PASJ, 37, 31

Shibata, K., \& Uchida, Y. 1986, PASJ, 38, 631

Sokolovsky, K. V., Kovalev, Y. Y., Pushkarev, A. B., \& Lobanov, A. P. 2011, A\&A, 532, A38

Tchekhovskoy, A., McKinney, J. C., \& Narayan, R. 2008, MNRAS, 388, 551

Tchekhovskoy, A., McKinney, J. C., \& Narayan, R. 2009, MNRAS, 699 , 1789

Uchida, Y., \& Shibata, K. 1985, PASJ, 37, 515

Uchida, Y., \& Shibata, K. 1986, Can. J. Phys., 64, 507

Vlahakis, N., Tsinganos, K., Sauty, C., \& Trussoni, E. 2000, MNRAS, 318, 417

Vlahakis, N. \& Königl, A. 2003a, ApJ, 596, 1080

Vlahakis, N. 2004, ApJ, 600, 324

Wang, C.-C., \& Zhou, H.-Y. 2009, MNRAS, 395, 301

Wilson, A. S., \& Yang, Y. 2002, ApJ, 568, 133

Wong, K.-W., et al. 2011, 736, L23

Young, A. J., Wilson, A. S., \& Mundell, C. G. 2002, ApJ, 579, 560

Yuan, F., Wu, M., \& Bu, D. 2012, ApJ, 2012, 761, 129

Yuan, F., Bu, D., \& Wu, M. 2012, ApJ, 2012, 761, 130

Zakamska, N. L., Begelman, M. C., \& Blandford, R. D. 2008, ApJ, 679, 990 